\title{
CKAP2 expression is associated with glioma tumor growth and acts as a prognostic factor in high-grade glioma
}

\author{
KUANYU WANG ${ }^{1,2^{*}}$, RUOYU HUANG ${ }^{1,2^{*}}$, GUANZHANG LI $^{1,2}$, FAN ZENG $^{2,3}$, \\ ZHENG ZHAO ${ }^{2,3}$, YANWEI LIU ${ }^{2,4}$, HUIMIN HU ${ }^{2,3}$ and TAO JIANG ${ }^{1,2,5}$ \\ ${ }^{1}$ Department of Neurosurgery, Beijing Neurosurgical Institute, Capital Medical University; \\ ${ }^{2}$ Chinese Glioma Cooperative Group (CGCG); ${ }^{3}$ Department of Neuropathology, Beijing Neurosurgical \\ Institute, Capital Medical University; Departments of ${ }^{4}$ Radiotherapy and ${ }^{5}$ Neurosurgery, \\ Beijing Tiantan Hospital, Capital Medical University, Beijing 100050, P.R. China
}

Received January 15, 2018; Accepted July 23, 2018

DOI: 10.3892/or.2018.6611

\begin{abstract}
Cytoskeletal-associated protein 2 (CKAP2), which is also known as tumor-associated microtubule-associated protein, has been reported to be dysregulated in various types of human cancer. However, the role of CKAP2 in glioma has not been fully elucidated. The present study evaluated the expression pattern of CKAP2 using the Chinese Glioma Genome Atlas microarray database, which included 301 patients, and validated the findings using The Cancer Genome Atlas RNA sequencing database. Kaplan-Meier survival analysis, and univariate and multivariate Cox analyses, were used to estimate survival distributions. Furthermore, the biological implication of aberrant CKAP2 expression in high-grade glioma (HGG) was investigated using Gene Ontology analysis, gene set enrichment analysis, gene set variation analysis and STRING. The results indicated that patients with HGG exhibited significantly higher CKAP2 expression levels compared with patients with low-grade glioma in both databases. Higher expression levels of CKAP2 were significantly associated with shorter overall survival and progression-free survival of patients with HGG. Furthermore, CKAP2 was also positively correlated with known malignant factors, including high Ki67 expression and phosphatase and tensin homolog mutations. The univariate and multivariate Cox regression analyses demonstrated that
\end{abstract}

Correspondence to: Dr Huimin Hu, Department of Neuropathology, Beijing Neurosurgical Institute, Capital Medical University, 6 Tiantan Xili, Beijing 100050, P.R. China

E-mail: huhm_bjni@163.com

Professor Tao Jiang, Department of Neurosurgery, Beijing Neurosurgical Institute, Capital Medical University, 6 Tiantan Xili, Beijing 100050, P.R. China

E-mail: taojiang1964@163.com

*Contributed equally

Key words: cytoskeletal-associated protein 2, glioma, histological type, tumor grade, cell cycle, prognosis
CKAP2 may be a novel independent prognostic biomarker for patients with HGG. Functional assays also indicated that CKAP2 was closely associated with the cell cycle, mitosis and cell proliferation. These results suggested that CKAP2 may be associated with tumor growth and could serve as an independent prognostic factor, particularly in patients with HGG.

\section{Introduction}

Glioma is the most common malignant tumor of the central nervous system $(1,2)$. Based on the World Health Organization (WHO) classification, gliomas are divided into four grades: WHO grade I-IV (3), in which WHO grade III (anaplastic glioma) and WHO grade IV [glioblastoma (GBM)] commonly constitute high-grade glioma (HGG), whereas WHO grade II is generally referred to as low-grade glioma (LGG) (4). HGG is highly lethal and aggressive, and complete removal is difficult, which ultimately leads to recurrence post-surgery. Despite advances in standard treatment, including surgical resection followed by radiotherapy and chemotherapy, satisfactory outcomes for patients with HGG are often limited (5). Compared with LGG, the migratory and invasive capability of HGG is more potent, and the doubling time of tumor cells is shorter. HGG tumor cells also have the ability to deeply infiltrate into adjacent normal brain tissue, which can result in incomplete resection, which is the main cause of postoperative recurrence. The occurrence and development of glioma is a multi-gene, multi-step and multi-stage process. Furthermore, various tumor-specific molecular alterations have been associated with glioma, in particular, isocitrate dehydrogenase 1 (IDH1) mutations (6), 1p/19q co-deletion (7), O6-methylguanine DNA methyltransferase (MGMT) promoter methylation (8) and epidermal growth factor receptor variant III amplification $(9,10)$ have been identified as predictive and prognostic indicators, and/or therapeutic targets for patients with glioma. It is necessary to identify novel biomarkers to provide more options for the treatment of patients with glioma.

Cytoskeletal-associated protein 2 (CKAP2), which is also known as tumor-associated microtubule-associated protein (TMAP), is localized on microtubule organizing centers and microtubules, and has an important role in cell mitosis (11). 
Previous research has revealed that CKAP2 is required for appropriate chromosome segregation and the maintenance of genomic stability $(12,13)$. CKAP2 was recently reported to be a substrate of cyclin-dependent kinase (CDK)1-cyclin B and was indicated to be essential for bipolar spindle formation (14). The oncogenic nature of CKAP2 in the occurrence and development of gastric (15), ovarian (16) and breast cancer (17), and other malignant tumors (18-21) has been elaborated. However, limited studies have assessed the biological characteristics and significance of CKAP2 in HGG.

The present study investigated the expression of CKAP2, and its association with the clinical features of glioma, using patient information from the Chinese Glioma Genome Atlas (CGGA) microarray database. The results demonstrated that the expression levels of CKAP2 were higher in patients with HGG compared with in patients with LGG. Subsequently, the patients with HGG were divided into two subgroups (cut off at $50 \%$ of the entire group) according to their CKAP2 expression levels; the survival analysis indicated that patients with HGG and lower CKAP2 expression levels exhibited a better prognosis. Subsequent studies using The Cancer Genome Atlas (TCGA) RNA sequencing database further validated these findings. Furthermore, univariate and multivariate Cox regression analyses demonstrated that high CKAP2 expression appeared to be a predictor for poor clinical outcomes in patients with HGG. Subsequently, CKAP2 positively correlated genes were analyzed using the Database for Annotation, Visualization and Integrated Discovery (DAVID) and gene set enrichment analysis (GSEA). In addition, Gene Ontology (GO) analysis indicated significant enrichment of the genes in the cell cycle, mitotic and cell proliferation pathways. In conclusion, the present study demonstrated that CKAP2 may be associated with glioma malignancy and could promote malignant progression by stimulating glioma cell proliferation. These characteristics of CKAP2 suggest that it may serve as a potential prognostic factor and a candidate gene for gene therapy of malignant glioma.

\section{Materials and methods}

CGGA microarray databases. A total of 301 patients with histologically confirmed glioma were enrolled in our study and their data was made available in the GGA database. Microarray data generated using the Agilent Whole Human Genome Array platform (Agilent Technologies, Santa Clara, CA, USA) (22) and follow-up information were obtained from all 301 patients. Following surgical resection, the histological diagnosis was performed independently by two experienced neuropathologists according to the 2016 WHO classification (3). During the study, none of the patients succumbed to other diseases or accidents. Written informed consent was obtained from all patients and the present study was approved by the ethics committee of Beijing Tiantan Hospital (Beijing, China). Details regarding the establishment and management of our CGGA database have been specified in our previous publication (23).

CKAP2 expression analysis in databases. A total of 301 glioma samples (122 grade II, 51 grade III and 128 grade IV samples) were obtained from the CGGA microarray database (http://www.cgga.org.cn/index. php?m=Page\&a=index\&id=42) as the discovery set. Data from TCGA RNA sequencing database (http://cancergenome.nih. gov; https://portal.gdc.cancer.gov/projects/TCGA-GBM and https://portal.gdc.cancer.gov/projects/TCGA-LGG), which contains 633 glioma samples (223 grade II, 242 grade III and 168 grade IV samples), were obtained as validation sets. TCGA RNA sequencing data were $\log 2$-transformed. In the two databases, only samples with definite WHO classification were included for expression analysis. Patients who were not clearly classified were excluded.

Cell transfection. Human astrocytes (HA; cat. no. 1800; ScienCell Research Laboratories, Inc., San Diego, CA, USA) cell line and human glioma cell lines H4, U-87MG ATCC (U87), LN229, U-118MG (U118) and U-251MG (U251) were purchased from the Institute of Biochemistry and Cell Biology, Chinese Academy of Sciences (Beijing, China). The CGGA-N33 cell line is a patient-derived GBM cell line established by the CGGA. Astrocyte Medium (cat. no. 1801; ScienCell Research Laboratories, Inc.) and Dulbecco's modified Eagle's medium (DMEM)/F12 (Gibco; Thermo Fisher Scientific, Inc., Waltham, MA, USA) supplemented with $10 \%$ fetal bovine serum (FBS; Gibco; Thermo Fisher Scientific, Inc.) were used to culture HA and CGGA-N33 cell lines, respectively. Human glioma cell lines were cultured in DMEM supplemented with $10 \%$ FBS. All cell lines were maintained in an incubator at $37^{\circ} \mathrm{C}$ in an atmosphere containing $5 \% \mathrm{CO}_{2}$. The following CKAP2 small interfering (si)RNA sequences were used in the present study: siRNA-1, 5'-GCACTACATCTCAGA ACAC-3'; siRNA-2, 5'-GGACTACCATGGCAGAAGA-3' and siRNA-3, 5'-GAGACGTTCTCGACGTCTT-3'. CKAP2 siRNA and negative control (NC; cat. no. siN05815122147) siRNA were synthesized by Guangzhou RiboBio Co., Ltd. (Guangzhou, China). Once cell density reached 30-50\%, U87 and CGGA-N33 cell lines were transfected with siRNA or $\mathrm{NC}(50 \mathrm{nM})$ at $37^{\circ} \mathrm{C}$ using the riboFECT CP Transfection kit (cat. no. C10511-1; Guangzhou RiboBio Co., Ltd.). After 48 h at $37^{\circ} \mathrm{C}$, fresh medium without siRNA was added to the cells.

Western blotanalysis. Total proteins were extracted using radioimmunoprecipitation assay buffer (Cell Signaling Technology, Inc., Danvers, MA, USA) supplemented with phenylmethylsulfonyl fluoride (1 mM; Beijing Solarbio Science \& Technology Co., Ltd., Beijing, China). Pierce Bicinchoninic Acid Protein Assay kit (Thermo Fisher Scientific, Inc.) was used to quantify protein concentration. The proteins were heated at $95-100^{\circ} \mathrm{C}$ with SDS-PAGE loading buffer (Beijing Solarbio Science \& Technology Co., Ltd.). Equal amounts of total protein $(20 \mu \mathrm{g})$ were separated by $10 \%$ SDS-PAGE and transferred to a polyvinylidene fluoride membrane (EMD Millipore, Billerica, MA, USA). The membrane was then blocked with 5\% skim milk (BD Biosciences, Franklin Lakes, NJ, USA) at room temperature for $1 \mathrm{~h}$. Subsequently, the membrane was incubated with primary antibodies at $4{ }^{\circ} \mathrm{C}$ overnight and with secondary antibodies at room temperature for $1 \mathrm{~h}$. The results were detected using an Enhanced Chemiluminescence Western Blotting Detection system (Bio-Rad Laboratories, Inc., Hercules, CA, USA). In the present study, western blot analysis was performed using rabbit anti-CKAP2 polyclonal antibody 
(1:800; cat. no. 25486-1-AP; Wuhan Sanying Biotechnology, Wuhan, China) and $\beta$-tubulin (1:5,000; cat. no. CW0098M; CWBIO, Beijing, China) was used as the loading control. Goat anti-rabbit and goat anti-mouse immunoglobulin G-horseradish peroxidase (1:5,000; cat. nos. ZB-2301 and ZB-2305; OriGene Technologies, Inc., Beijing, China,) were used as secondary antibodies to detect CKAP2 and $\beta$-tubulin, respectively.

RNA extraction and reverse transcription-quantitative polymerase chain reaction $(R T-q P C R)$. Post-transfection with CKAP2 and NC siRNA, total RNA was extracted from U87 and CGGA-N33 glioma cells using RNAprep Pure kit for cell/bacteria (Tiangen Biotech Co., Ltd., Beijing, China). RNA was stored at $-80^{\circ} \mathrm{C}$ and was then reverse transcribed using the RevertAid First Strand cDNA Synthesis kit (Thermo Fisher Scientific, Inc), according to the manufacturer's protocol. To detect alterations in the relative mRNA expression levels of CKAP2 post-transfection with specific siRNA, RT-qPCR was performed using the SYBR SuperMix kit (Bio-Rad Laboratories, Inc.) and the 7500 Fast Real-Time PCR system (Applied Biosystems, USA), according to the manufacturer's protocols. The thermocycling conditions were conducted according to the manufacturer's protocol, as follows: i) Uracil DNA glycosylase activation at $50^{\circ} \mathrm{C}$ for $2 \mathrm{~min}$; ii) $95^{\circ} \mathrm{C}$ for $2 \mathrm{~min}$; iii) 40 cycles at $95^{\circ} \mathrm{C}$ for $15 \mathrm{sec}$ and $60^{\circ} \mathrm{C}$ for $1 \mathrm{~min}$. Fluorescence measurements were taken at each cycle. The relative mRNA expression levels of CKAP2 were normalized to GAPDH and were calculated using the $2^{-\Delta \Delta \mathrm{Cq}}$ method (24). The primer sequences of CKAP2 and GAPDH endogenous control were purchased from GENEWIZ (Beijing, China). The primer sequences were as follows: CKAP2, forward 5'-GCA AGATGCTAACATGCCCAA-3', and reverse 5'-TGGCTT TAGGTATAGTGGCTGA-3'; and GAPDH, forward 5'-GGA GCGAGATCCCTCCAAAAT-3', and reverse 5'-GGCTGT TGTCATACTTCTCATGG-3'.

Clonogenic assay. U87 and CGGA-N33 glioma cells in culture plates were digested with $0.25 \%$ trypsin-EDTA (Gibco; Thermo Fisher Scientific, Inc.) and dissociated. Subsequently, cells were seeded into 12-well culture plates (200 cells/well). Following adherence, cells were transfected with CKAP2 or NC siRNA and the medium was replaced after $12 \mathrm{~h}$. Plates were incubated at $37^{\circ} \mathrm{C}$ in an atmosphere containing $5 \% \mathrm{CO}_{2}$ for 12 days before the cell colonies were stained with $0.2 \%$ crystal violet at room temperature for $5 \mathrm{~min}$. An inverted fluorescence microscope (Carl Zeiss AG, Oberkochen, Germany) was used for in vitro analysis.

Bioinformatics analysis. Pearson's correlation analysis was performed using $\mathrm{R}$ language 3.2.5 (https://cran.r-project. org/bin/windows/base/old/3.2.5/) to determine the correlation between CKAP2 and other genes in the CGGA microarray database and TCGA RNA sequencing database. Positively correlated CKAP2 genes $(r>0.4, \mathrm{P}<0.01)$ were analyzed using DAVID (http://david.abcc.ncifcrf.gov/home.jsp) and GSEA to detect the association between biological processes and CKAP2 expression in glioma. GO and Kyoto Encyclopedia of Genes and Genomes pathway enrichment analyses, conducted using DAVID, were the main methods of bioinformatics analysis used in the present study. Gene set variation analysis (GSVA) with CKAP2 was analyzed using a GSVA package
Table I. Clinical characteristics of 301 patients with glioma in the Chinese Glioma Genome Atlas database.

\begin{tabular}{lcc}
\hline Variable & $\begin{array}{c}\text { Low-grade } \\
\text { glioma }^{\mathrm{a}}\end{array}$ & $\begin{array}{c}\text { High-grade } \\
\text { glioma }\end{array}$ \\
\hline Number & 122 & 179 \\
Gender, male/female & $71 / 51$ & $109 / 70$ \\
Median age (range), years & $37.5(18-61)$ & $45(13-70)$ \\
IDH1 wild-type & 36 & 129 \\
IDH1 mutation & 82 & 50 \\
\hline
\end{tabular}

${ }^{a}$ IDH1 expression data were not available for four patients with low-grade glioma. IDH1, isocitrate dehydrogenase 1.

of $\mathrm{R}$ (25). The list of CKAP2-related functions and pathways in GSVA were obtained from the GO database website (http://amigo.geneontology.org/amigo). Protein-protein interactions of CKAP2 were analyzed using the STRING v10.0 online tool (https://string-db.org/).

Statistical analysis. Statistical analysis was performed using SPSS 16.0 (SPSS, Inc., Chicago, IL, USA), R language 3.2.5 and GraphPad Prism 7.0 statistical software (GraphPad Software, Inc., La Jolla, CA, USA). Student's t-test was used to compare the difference in expression levels between patients with HGG and LGG. One-way analysis of variance followed by Holm-Sidak test was used to compare the difference in CKAP2 expression between various histological grades and molecular subtypes of glioma; the RT-qPCR results were also analyzed in this manner. Kaplan-Meier (K-M) survival analysis with long-rank test was used to assess the predictive value of CKAP2 expression for overall survival (OS) in the two databases and progression-free survival (PFS) in the CGGA microarray database between various grades of gliomas. In the K-M survival analysis, the patients with HGG were divided into two subgroups (cut off at $50 \%$ of the entire group) according to the expression levels of CKAP2. OS was calculated from the data obtained from histological diagnosis until mortality, whereas PFS was calculated until tumor recurrence, which was diagnosed by magnetic resonance imaging. Univariate and multivariate Cox regression analyses, including age at diagnosis, gender, IDH1 mutation status and CKAP2 expression were used to assess the prognostic value of CKAP2 in HGG. A two-sided $\mathrm{P}<0.05$ was considered to indicate a statistically significant difference.

\section{Results}

Baseline clinical characteristics. The clinical and microarray data were obtained from 301 patients with glioma in the CGGA microarray database, including 122 patients with LGG (WHO grade II) and 179 patients with HGG (49 patients with WHO grade III glioma and 130 patients with GBM). The baseline characteristics of all patients are summarized in Table I. In TCGA RNA sequencing database, data from a total of 636 patients, including 223 patients with LGG and 413 patients with HGG were obtained. The expression levels of 
A
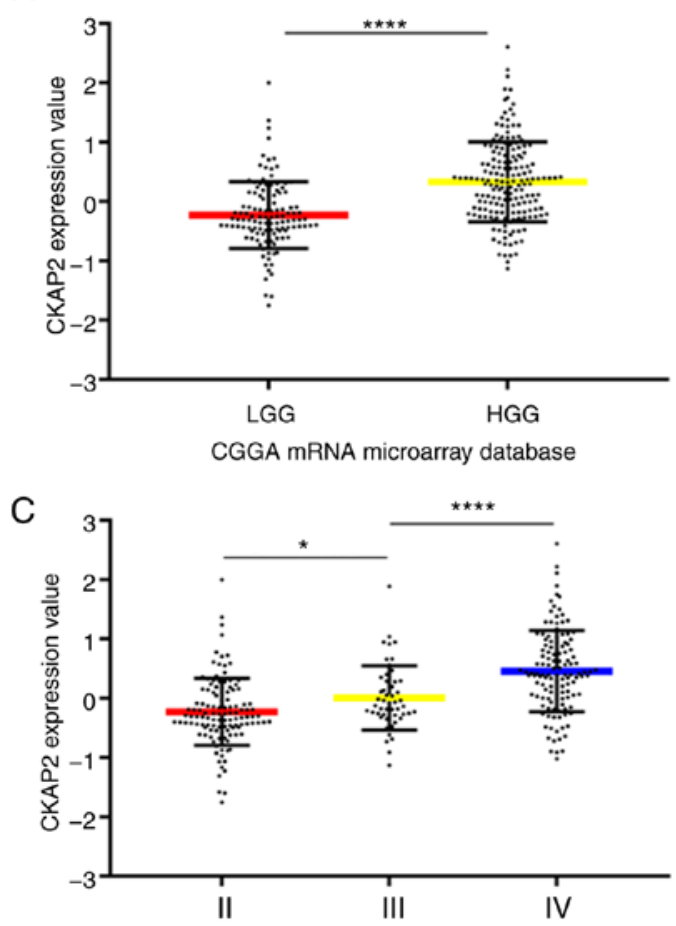

CGGA mRNA microarray database

E

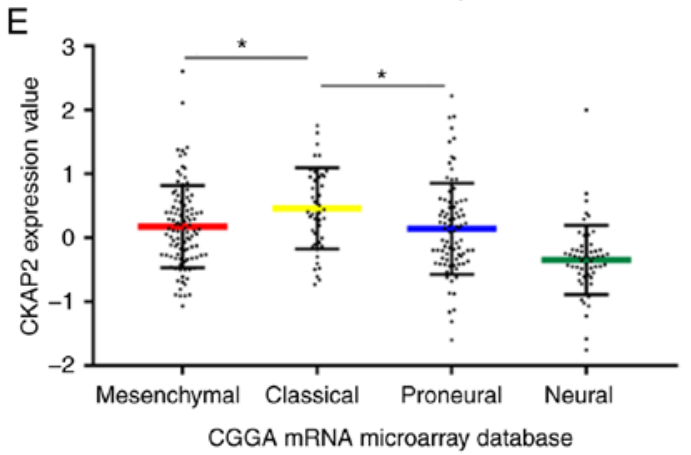

B

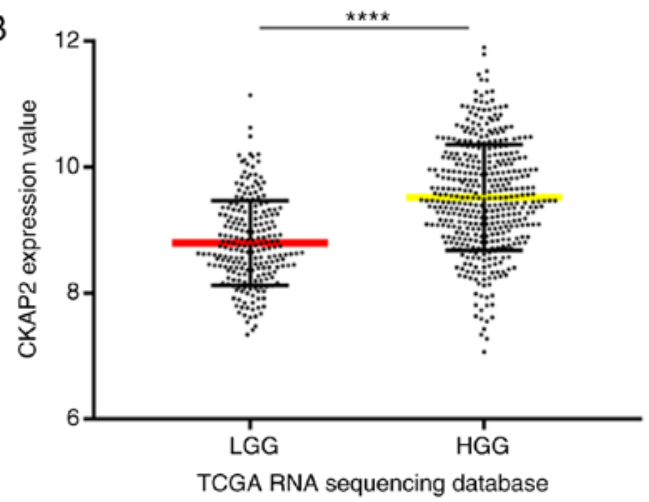

D

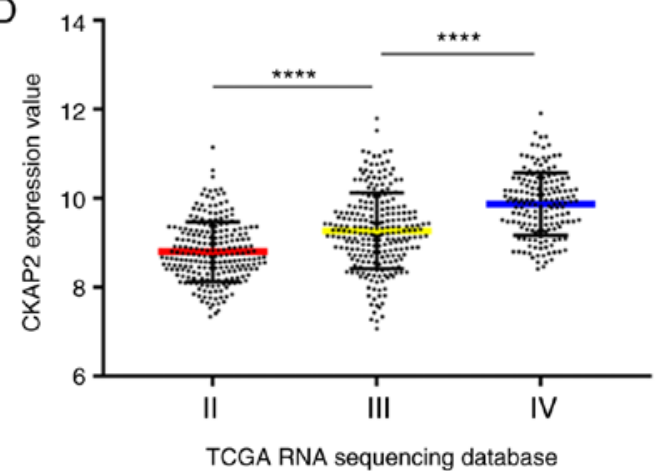

$\mathrm{F}$

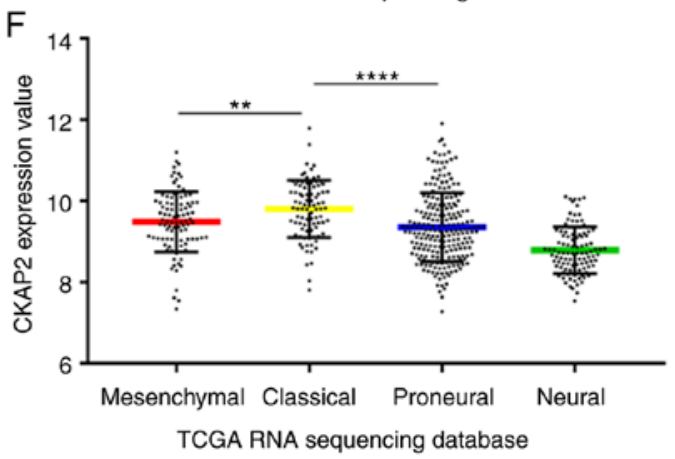

Figure 1. CKAP2 mRNA expression in tumor samples with different grades and subtypes in the CGGA and TCGA databases. (A and B) mRNA expression levels of CKAP2 were higher in HGG patient samples compared with in LGG patient samples in the CGGA and TCGA databases. (C and D) mRNA expression levels of CKAP2 from patient samples obtained from the CGGA and TCGA databases were positively associated with tumor grade. (E and F) CKAP2 expression was highest in classical subtype glioma samples. ${ }^{*} \mathrm{P}<0.05,{ }^{* *} \mathrm{P}<0.01,{ }^{* * * * *} \mathrm{P}<0.0001$. CGGA, Chinese Glioma Genome Atlas; CKAP2, cytoskeletal-associated protein 2; HGG, high-grade glioma; LGG, low-grade glioma; TCGA, The Cancer Genome Atlas.

CKAP2 and the clinical characteristics of patients were used for validation analysis.

Expression of CKAP2 in gliomas of different grades and subtypes. The present study screened for the differentially expressed genes between LGG and HGG in the CGGA microarray database and demonstrated that CKAP2 was significant. Compared with in patients with LGG, the mRNA expression levels of CKAP2 were significantly higher in HGG patient samples (Fig. 1A). Subsequently, the mRNA expression levels of CKAP2 were analyzed in patients with glioma in TCGA RNA sequencing database. Similarly, CKAP2 mRNA expression was significantly higher in patients with HGG than in patients with LGG (Fig. 1B). Furthermore, after the differential expression of CKAP2 was screened from all patients from the CGGA microarray database and TCGA RNA sequencing database, it was demonstrated that CKAP2 expression was positively associated with tumor grade (Fig. 1C and D). In the CGGA database, CKAP2 mRNA expression levels were significantly different in four molecular subtypes of glioma, as defined by a TCGA network. Compared with the other subtypes, the classical subtype primarily exhibited the higher CKAP2 expression (Fig. 1E); as validation, analysis of TCGA database exhibited similar results (Fig. 1F). These results suggested that CKAP2 may have the potential to serve as a biomarker for the classical subtype of glioma.

Association between CKAP2 mRNA expression and clinical outcomes. The association between CKAP2 mRNA expression and the clinical outcomes of patients with HGG was investigated by K-M survival curve analysis, using data from 301 patients with glioma from the CGGA microarray database and 633 patients with glioma from TCGA RNA sequencing database. Patients with HGG were divided into two subgroups (cut off at $50 \%$ of the entire group) according to CKAP2 expression levels. K-M analysis indicated that there 
A

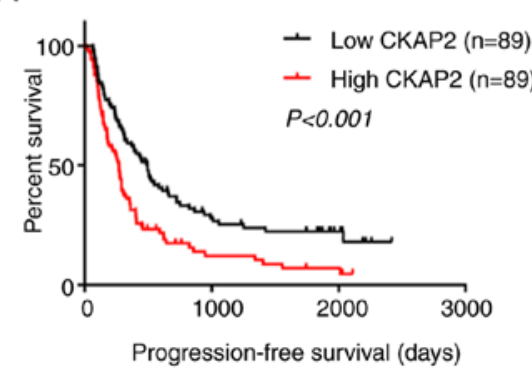

B

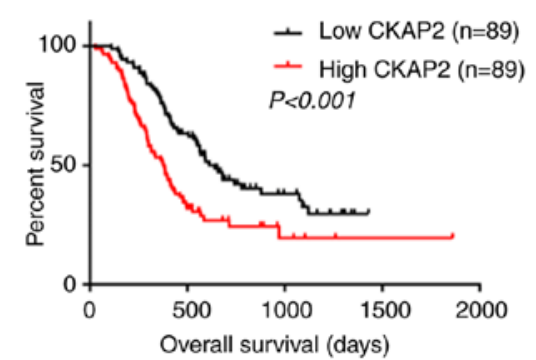

C

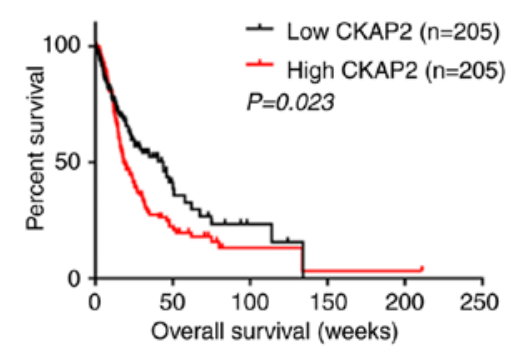

G CGGA mRNA microarray database, GBM

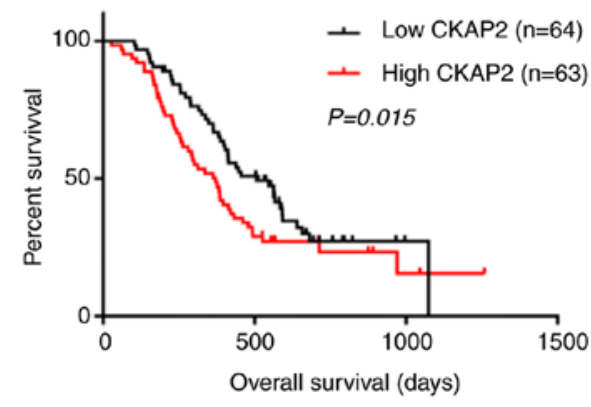

I
D CGGA mRNA microarray database,LGG

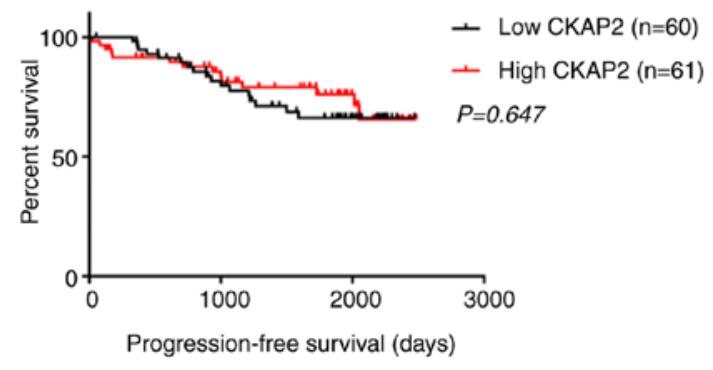

E

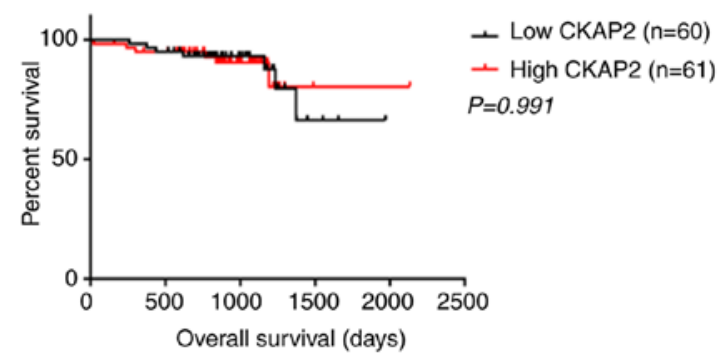

F TCGA RNA sequencing database, LGG

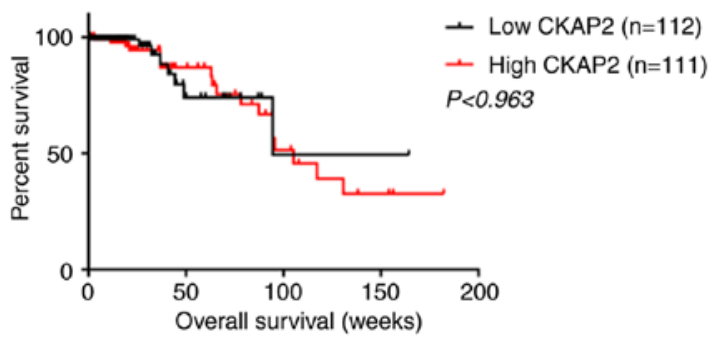

H CGGA mRNA microarray database, GBM

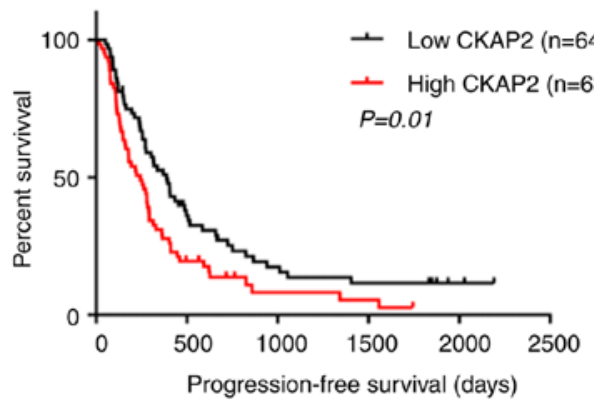

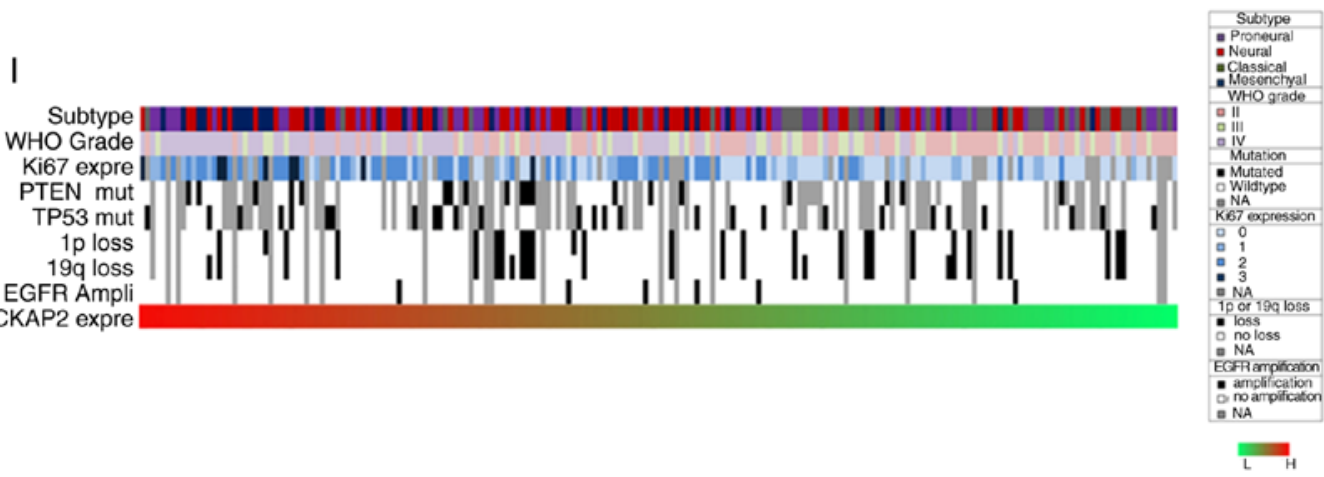

Figure 2. High CKAP2 expression is associated with poor prognosis in patients with HGG. (A and B) PFS and OS of patients with HGG from the CGGA database separated into high and low CKAP2 expression groups. (C) OS of patients with HGG from TCGA database separated into high and low CKAP2 expression groups. (D and E) PFS and OS of patients with LGG from the CGGA database separated into CKAP2 high and low expression groups. (F) OS of patients with LGG from TCGA database separated into CKAP2 high and low expression groups. (G and H) OS and PFS of patients with GBM from the CGGA database separated into high and low CKAP2 expression groups. (I) Correlation of CKAP2 expression with well-known genomic alterations in glioma. Ampli, amplification; CGGA, Chinese Glioma Genome Atlas; CKAP2, cytoskeletal-associated protein 2; expre, expression; H, high; HGG, high-grade glioma; L, low; LGG, low-grade glioma; mut, mutation; OS, overall survival; PFS, progression-free survival; TCGA, The Cancer Genome Atlas. 
Table II. Univariate and multivariate analysis of overall survival in the Chinese Glioma Genome Atlas microarray database.

\begin{tabular}{|c|c|c|c|c|}
\hline \multirow[b]{2}{*}{ Variable } & \multicolumn{2}{|c|}{ Univariate analysis } & \multicolumn{2}{|c|}{ Multivariate analysis } \\
\hline & HR $(95 \%$ CI $)$ & P-value & HR $(95 \%$ CI $)$ & P-value \\
\hline CKAP2 expression & $1.530(1.160-2.018)$ & 0.003 & $1.704(1.027-2.827)$ & 0.039 \\
\hline Age & $1.018(1.002-1.034)$ & 0.031 & $0.987(0.961-1.013)$ & 0.325 \\
\hline Gender & $1.186(0.813-1.732)$ & 0.376 & & \\
\hline Grade & $2.266(1.435-3.579)$ & $<0.001$ & $0.983(0.425-2.274)$ & 0.968 \\
\hline KPS & $0.968(0.953-0.983)$ & $<0.001$ & $0.959(0.942-0.976)$ & $<0.001$ \\
\hline IDH1 mutation & $0.550(0.353-0.855)$ & 0.008 & $0.539(0.239-1.214)$ & 0.136 \\
\hline MGMT methylation & $0.572(0.381-0.858)$ & 0.007 & $0.499(0.246-1.012)$ & 0.054 \\
\hline Chemotherapy & $0.449(0.307-0.657)$ & $<0.001$ & $0.550(0.292-1.037)$ & 0.065 \\
\hline Radiotherapy & $0.414(0.259-0.664)$ & $<0.001$ & $0.607(0.282-1.308)$ & 0.203 \\
\hline
\end{tabular}

CI, confidence interval; CKAP2, cytoskeletal-associated protein 2; HR, hazard ratio; IDH1, isocitrate dehydrogenase 1; KPS, Karnofsky Performance Status score; MGMT, O6-methylguanine DNA methyltransferase.

Table III. Univariate and multivariate analysis of progression-free survival in the Chinese Glioma Genome Atlas microarray database.

\begin{tabular}{|c|c|c|c|c|}
\hline \multirow[b]{2}{*}{ Variables } & \multicolumn{2}{|c|}{ Univariate analysis } & \multicolumn{2}{|c|}{ Multivariate analysis } \\
\hline & HR (95\% CI) & P-value & HR $(95 \% \mathrm{CI})$ & P-value \\
\hline CKAP2 Expression & $1.486(1.171-1.884)$ & 0.001 & $2.003(1.207-3.324)$ & 0.007 \\
\hline Age & $1.021(1.007-1.035)$ & 0.003 & $0.990(0.966-1.015)$ & 0.430 \\
\hline Gender & $1.114(0.798-1.555)$ & 0.526 & & \\
\hline Grade & $1.927(1.307-2.842)$ & 0.001 & $0.662(0.300-1.461)$ & 0.307 \\
\hline KPS & $0.978(0.965-0.992)$ & 0.002 & $0.971(0.965-0.987)$ & $<0.001$ \\
\hline IDH1 mutation & $0.546(0.370-0.807)$ & 0.002 & $0.583(0.276-1.232)$ & 0.158 \\
\hline MGMT methylation & $0.617(0.430-0.887)$ & 0.009 & $0.531(0.270-1.044)$ & 0.067 \\
\hline Chemotherapy & $0.448(0.317-0.633)$ & $<0.001$ & $0.580(0.323-1.042)$ & 0.068 \\
\hline Radiotherapy & $0.600(0.385-0.935)$ & 0.024 & $0.909(0.437-1.891)$ & 0.799 \\
\hline
\end{tabular}

CI, confidence interval; CKAP2, cytoskeletal-associated protein 2; HR, hazard ratio; IDH1, isocitrate dehydrogenase 1; KPS, Karnofsky Performance Status score; MGMT, O6-methylguanine DNA methyltransferase.

was a significant difference in PFS $(\mathrm{P}<0.001$, log-rank test; Fig. 2A) and OS (P<0.001, log-rank test; Fig. 2B) between high and low CKAP2 expression groups in the CGGA microarray database. Patients with HGG and high CKAP2 mRNA expression were observed to have a worse prognosis compared with those with low CKAP2 expression. In TCGA RNA sequencing database, the patients with HGG and high CKAP2 expression also exhibited worse OS compared with those with low CKAP2 expression ( $\mathrm{P}=0.023$, log-rank test; Fig. 2C). Conversely, neither PFS nor OS were significantly altered in patients with LGG according to CKAP2 expression, as determined using the CGGA microarray database $(\mathrm{P}=0.647$ and 0.991 , respectively, log-rank test; Fig. 2D and E) and TCGA RNA sequencing database $(\mathrm{P}=0.963$, log-rank test; Fig. $2 \mathrm{~F})$. Taken together, these results indicated that CKAP2 expression may serve as a prognostic factor for patients with HGG. In consideration of the heterogeneity across different glioma grades, the present study further investigated the prognostic value of CKAP2 expression in patients with GBM from the CGGA database $(\mathrm{P}=0.015$ and $0.01, \mathrm{OS}$ and $\mathrm{PFS}$, respectively, log-rank test; Fig. $2 \mathrm{G}$ and $\mathrm{H}$ ) and observed a similar pattern according to the K-M curves.

After exploring CKAP2-associated genomic alterations, an overview of the associations between CKAP2 expression and well-known genomic or transcriptional alterations in glioma was obtained (Fig. 2I). According to these results, the incidence of the biomarkers that indicate a poor prognosis, including high Ki67 expression and phosphatase and tensin homolog mutations, were higher in patients with glioma and higher CKAP2 expression. These findings further confirmed that CKAP2 expression was associated with poor clinical outcomes.

CKAP2 is a novel independent prognostic biomarker for patients with HGG. Univariate Cox regression analysis was 
A

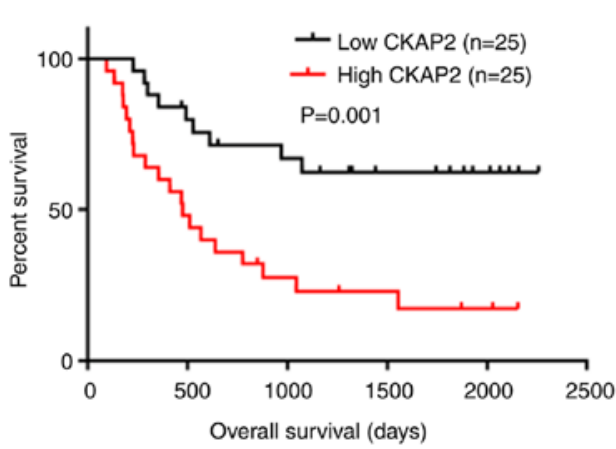

B

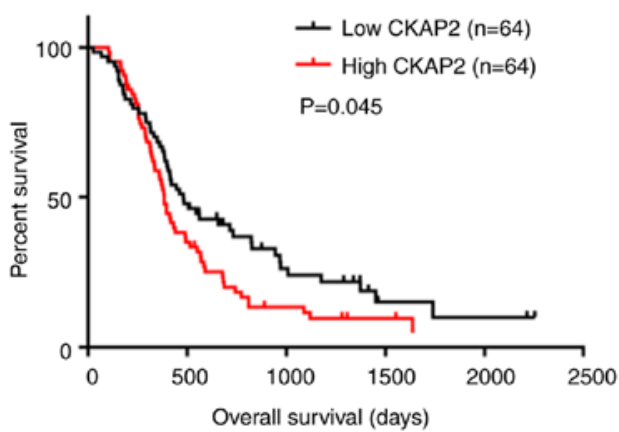

Figure 3. Kaplan-Meier survival curve analysis of patients with HGG and different IDH1 mutation states based on CKAP2 expression. (A) OS of patients with HGG and IDH1 mutations separated into high and low CKAP2 expression groups. (B) OS of patients with HGG and wild-type IDH1 separated into high and low CKAP2 expression groups. CKAP2, cytoskeletal-associated protein 2; H, high; HGG, high-grade glioma; IDH1, isocitrate dehydrogenase 1; OS, overall survival.
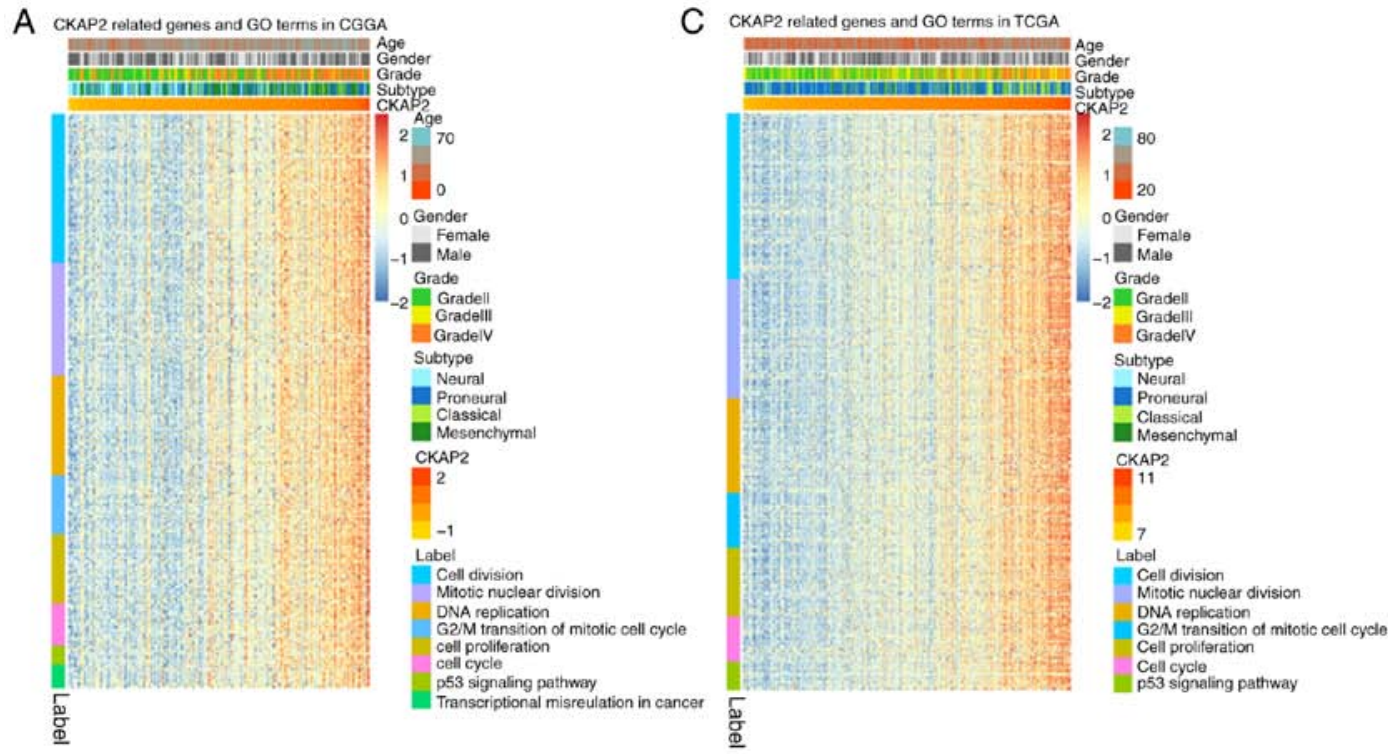

B
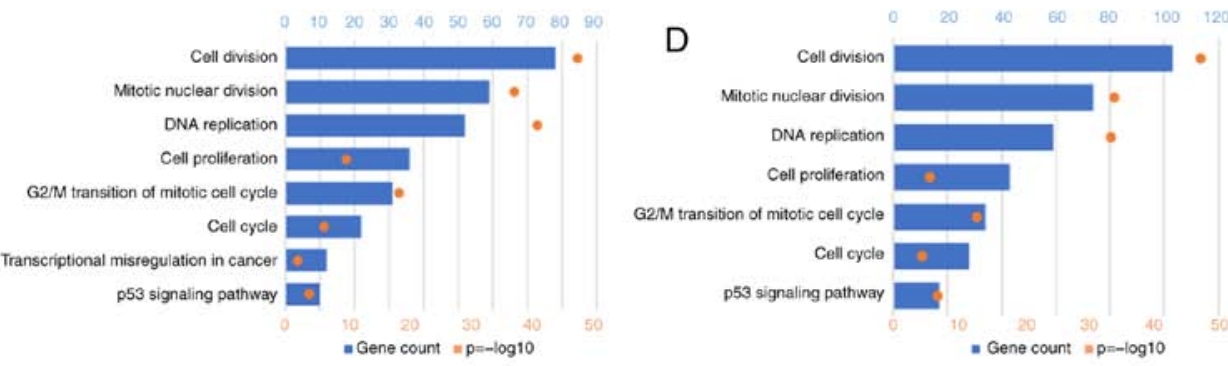

$\mathrm{E}$
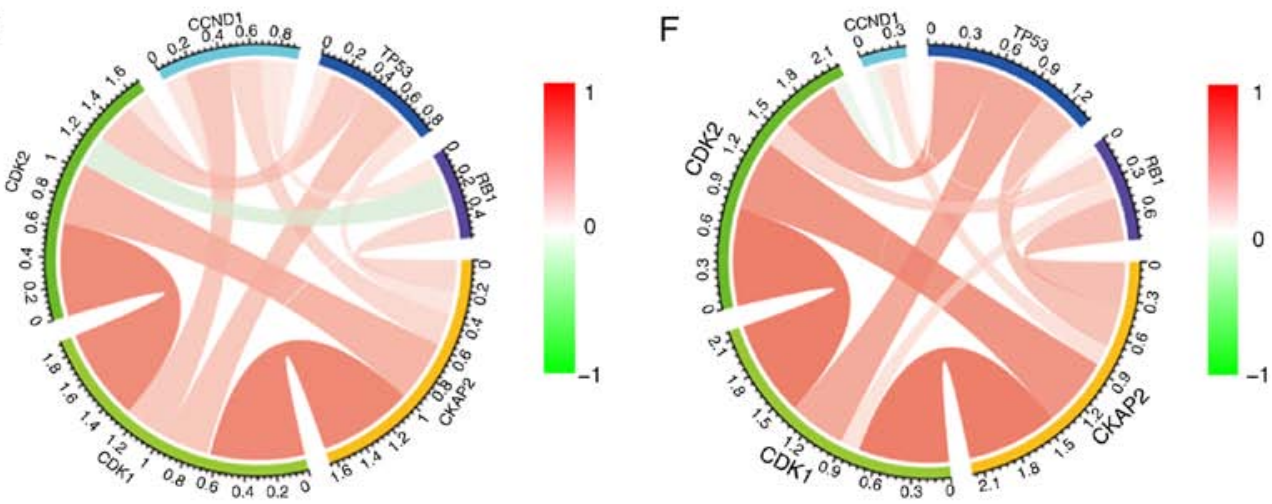

Figure 4. GO analysis of CKAP2 and association with cell cycle checkpoint members in HGG. (A and B) GO analysis of CKAP2-associated genes in HGG samples from the CGGA database. (C and D) GO analysis of CKAP2-associated genes in HGG samples from TCGA database. (E and F) Association between CKAP2 and cell cycle checkpoint members in HGG samples from the CGGA and TCGA databases. CGGA, Chinese Glioma Genome Atlas; CKAP2, cytoskeletal-associated protein 2; HGG, high-grade glioma; GO, Gene Ontology; TCGA, The Cancer Genome Atlas. 

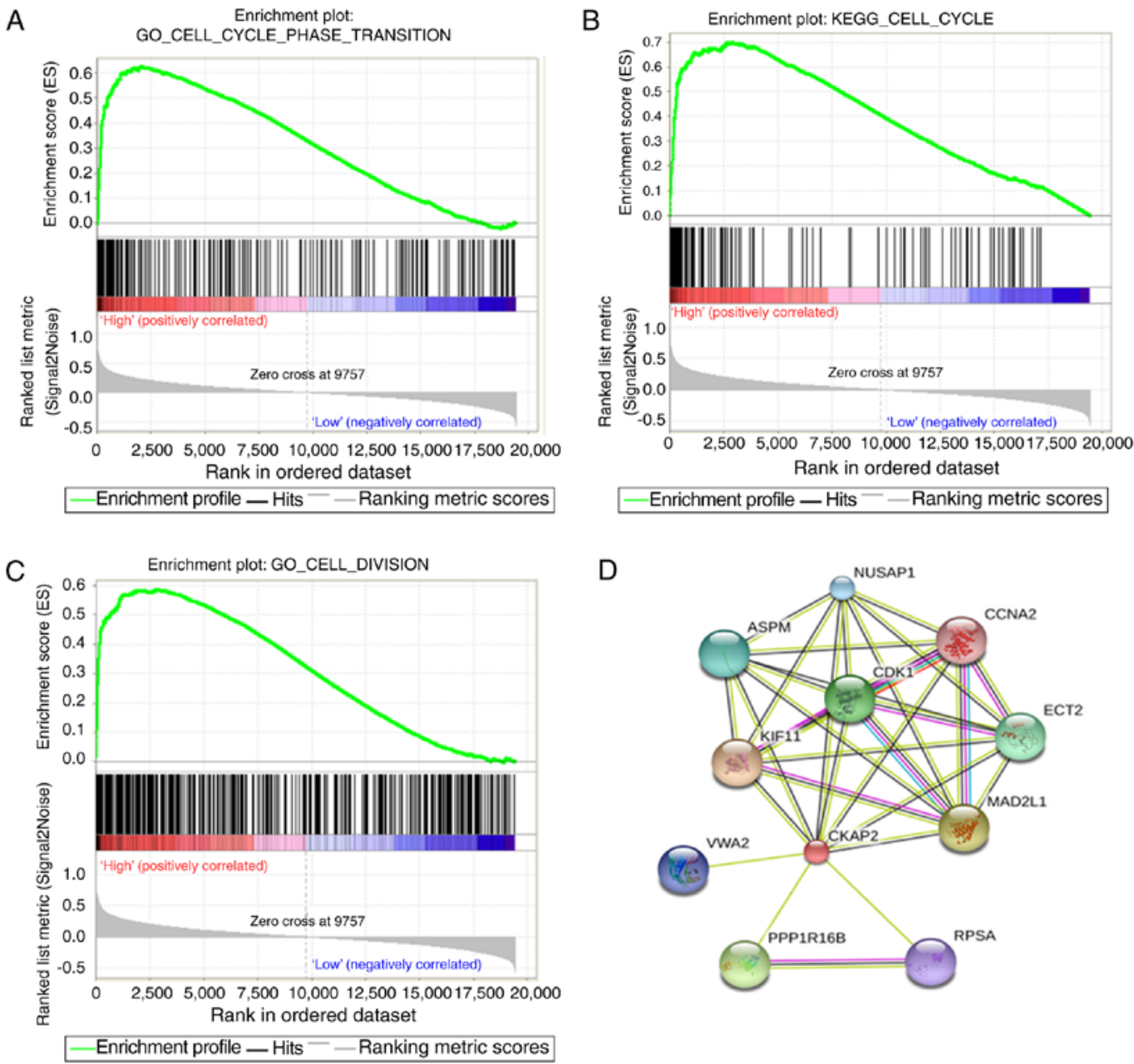

Figure 5. (A-C) Gene Ontology and Kyoto Encyclopedia of Genes and Genomes pathway enrichment analyses in the high CKAP2 expression group of patients with glioma from the Chinese Glioma Genome Atlas database were analyzed by gene set enrichment analysis. (D) Protein-protein interactions of CKAP2 were analyzed using STRING. CKAP2, cytoskeletal-associated protein.

performed on data obtained from the CGGA database to further determine the value of CKAP 2 and other variables in predicting OS and PFS of patients with HGG (Tables II and III). The results demonstrated that high $\mathrm{CKAP} 2$ expression was a risk factor for patients with $\mathrm{HGG}(\mathrm{P}=0.003$ for $\mathrm{OS}$ and $\mathrm{P}=0.001$ for PFS). Furthermore, other factors, including age and IDH1 mutation state, were also significantly associated with the OS and PFS of patients with HGG. A multivariate Cox regression analysis was performed incorporating CKAP2 expression, age, WHO grade, Karnofsky Performance Status score, MGMT methylation state, IDH1 mutation state, chemotherapy and radiotherapy (Tables II and III). The results revealed that CKAP2 was an independent predictive factor for the OS and PFS of patients with HGG $(\mathrm{P}=0.039$ for $\mathrm{OS}$ and $\mathrm{P}=0.007$ for PFS). In addition, the results of K-M survival curve analysis indicated that high expression levels of CKAP2 were closely associated with poor prognosis in patients with HGG with or without IDH1 mutations $(\mathrm{P}=0.001$ for IDH1 mutation and $\mathrm{P}=0.045$ for IDH1 wild-type, log-rank test; Fig. 3A and B).

CKAP2 is associated with the cell cycle, mitosis and cell proliferation. To identify the association between CKAP2 expression and other genes in glioma, Pearson's correlation analysis was used on data obtained from the CGGA and TCGA databases. The results demonstrated that 560 genes in the CGGA database and 805 genes in TCGA database were positively correlated with CKAP2 ( $>0.4, \mathrm{P}<0.01)$. Subsequently, these genes were subjected to GO analysis. The top GO terms indicated that CKAP2 was significantly associated with gene sets associated with the cell cycle, mitotic nuclear division and cell proliferation (Fig. 4A-D). Circos plots demonstrated that CKAP2 expression was closely associated with CDK1 and CDK2 (Fig. 4E and F), both of which serve vital roles in the cell cycle and cell proliferation. GSEA results were similar to those obtained from GO analysis (Fig. 5A-C). The protein-protein interactions of CKAP2 analyzed by STRING indicated that CKAP2 was closely associated with kinesin family member 11 , CDK1 and mitotic arrest deficient 2 like 1 (Fig. 5D). Notably these three genes have been identified to serve a vital role in the cell cycle and cell mitosis (26-28). Furthermore, by applying GSVA on CGGA and TCGA data, the previously reported cell cycle-, mitotic- and cell proliferation-associated genes also exhibited a higher enrichment score in the high-risk group (Fig. 6A and B). These analyses indicated that CKAP2 may have an essential role in the cell cycle, mitosis and proliferation of glioma cells.

CKAP2 promotes the proliferation of glioma cells in vitro. As indicated by GO analysis, the overexpression of CKAP2 
A

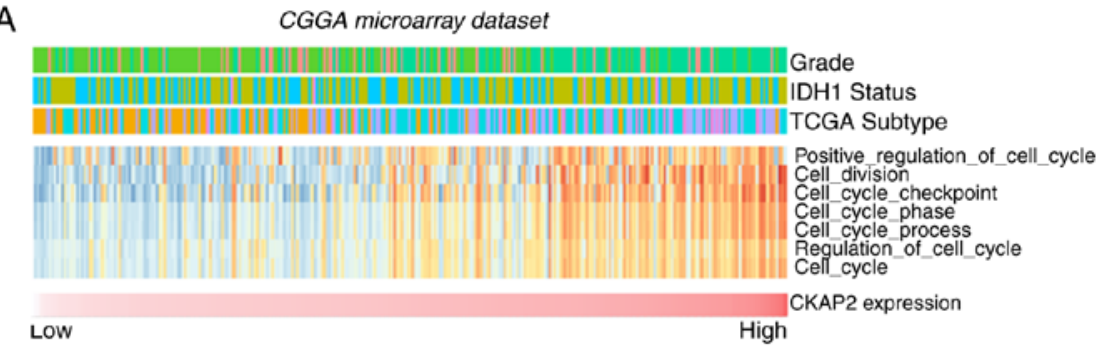

B

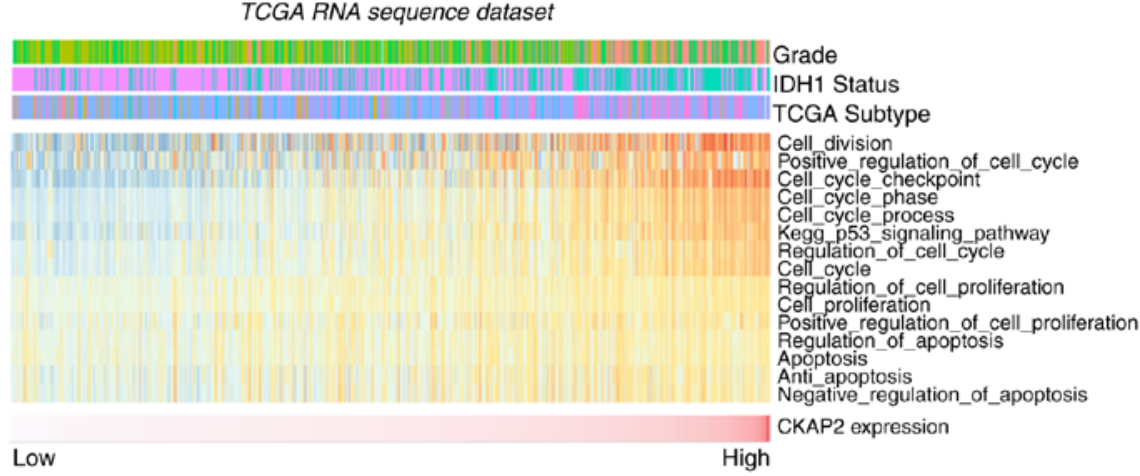

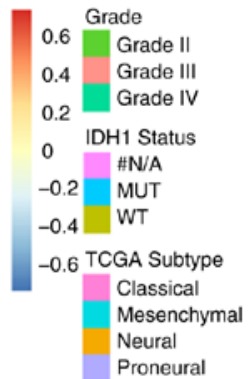

\begin{tabular}{|l|l}
\multicolumn{2}{c}{ Grade } \\
0.5 & $\begin{array}{l}\text { Grade II } \\
\text { Grade III } \\
\text { Grade IV }\end{array}$
\end{tabular}

IDH Status

Mutant

$-0.5$ WT

Figure 6. (A and B) Gene set variation analysis of CKAP2 in glioma samples from the CGGA and TCGA database. CGGA, Chinese Glioma Genome Atlas; CKAP2, cytoskeletal-associated protein 2; IDH, isocitrate dehydrogenase; IDH1, isocitrate dehydrogenase 1; TCGA, The Cancer Genome Atlas.
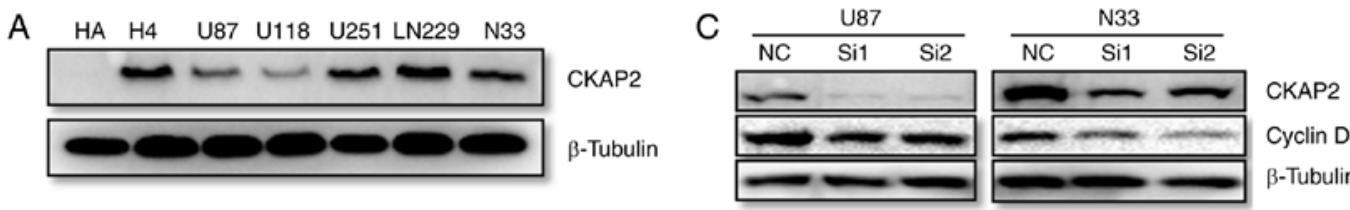

Cyclin D1 $\beta$-Tubulin
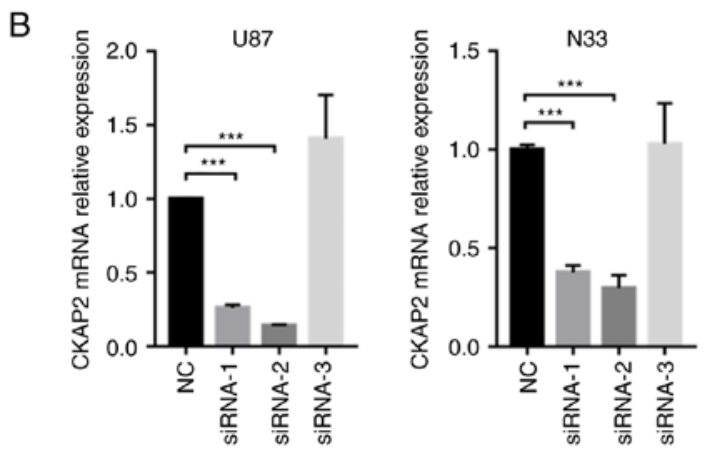

D
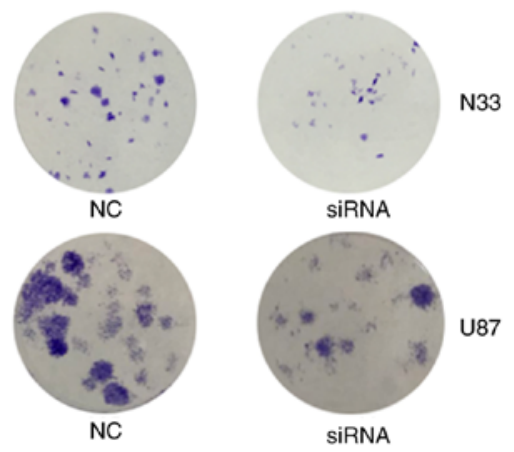

Figure 7. Carcinogenic effects of CKAP2 on glioma cell lines. (A) CKAP2 protein expression was evaluated in HA and glioma cell lines. (B) CKAP2 mRNA expression was downregulated by CKAP2 siRNA-1 and siRNA-2 in U87 and CGGA-N33 cells. (C) Protein expression levels of CKAP2 and cyclin D1 were decreased post-transfection of U87 and CGGA-N33 cells with CKAP2 siRNA. (D) Results of a clonogenic assay indicated that knockdown of CKAP2 expression suppressed glioma cell proliferation. CKAP2, cytoskeletal-associated protein 2; HA, human astrocytes; NC, negative control; siRNA, small interfering RNA.

was positively associated with cell division and cell proliferation. To further determine the functional roles of CKAP2 in glioma, a series of in vitro experiments were performed. The protein expression levels of CKAP2 were initially detected in H4, LN229, U87, U251, U118 and CGGA-N33 glioma cell lines, and the HA cell line. Western blot analysis indicated that all glioma cells exhibited a higher CKAP2 expression compared with HA (Fig. 7A). In addition, CKAP2 siRNA was transfected to knockdown CKAP2 expression in U87 and CGGA-N33 cells. RT-qPCR demonstrated that the mRNA expression levels of CKAP2 were significantly decreased in
U87 and CGGA-N33 cells post-transfection with siRNA-1 and siRNA-2 (Fig. 7B). Furthermore, western blot analysis indicated that the protein expression levels of the cell mitotic biomarker cyclin D1 were also significantly decreased in siRNA-transfected U87 and CGGA-N33 cells (Fig. 7C). These findings indicated that CKAP2 may have a significant impact on cell division. Additionally, to evaluate the function of CKAP2 in glioma cell lines, a clonogenic assay was performed. The results indicated that silencing CKAP2 expression suppressed the proliferative capacity and clonogenicity of glioma cells (Fig. 7D). 


\section{Discussion}

HGG refers to WHO grade III and IV glioma (4). HGG is highly malignant and inevitably recurs following surgical resection, due to its highly invasive behavior; therefore, patients with HGG have a typically poor outcome (29). Therefore, there is an urgent requirement to understand the mechanism of occurrence and development of this disease and to identify useful prognostic biomarkers for the OS of patients with HGG. Tumor growth is a complex and multistep process; during this process, the cell cycle, mitosis and cell proliferation all have crucial roles and are influenced by numerous regulators.

CKAP2, also known as TMAP, serves a vital role in cell mitosis and cell death in a P53-dependent manner (30). Previous research has been performed on various human tumors, including gastric cancer, breast cancer, ovarian cancer and prostate cancer (15-21). Notably, CKAP2 has been reported to be overexpressed in the aforementioned cancer types and reduces the OS of patients by various mechanisms. For example, gastric adenocarcinoma, which is a poorly differentiated, highly malignant adenocarcinoma, possesses a relatively high number of cytoplasmic CKAP2-positive cells (15). In addition, in ovarian cancer, the OS of patients with high levels of CKAP2 is shorter, and CKAP2, along with butyrylcholinesterase, claudin 10 and oviductal glycoprotein 1 mediates resistance to chemotherapy (16). In a previous study of early-stage breast cancer, high CKAP2 expression was revealed to promote the proliferation of tumor cells and significantly shorten relapse-free survival (17). Additionally, overexpression of CKAP2 activates the focal adhesion kinase (FAK)-extracellular signal-regulated kinase 2 signaling pathway, and induces cell proliferation and migration; however, this effect can be blocked by FAK inhibitors (18). In addition to CKAP2, other members of the CKAP family have also demonstrated similar effects on tumor cells, including CKAP4. Notably, after binding to Dickkopf 1, CKAP4 forms a complex with phosphoinositide 3-kinase (PI3K) and leads to activation of the PI3K-protein kinase B signaling pathway and tumor cell proliferation (31). These previous findings suggested that CKAP2 may potentially have an important role in the progression of cancer.

The expression levels and functions of CKAP2 in glioma have not been fully elucidated. To the best of our knowledge, the present study is the first to investigate CKAP2 expression in a large number of patients with glioma. The retrospective study was performed to evaluate the expression of CKAP2 and the clinical data of 934 patients with glioma in the CGGA microarray database and TCGA RNA sequencing database. The results demonstrated that the expression levels of CKAP2 were closely associated with glioma grade. Furthermore, in both databases, the OS of patients with HGG and high CKAP2 expression was shorter. Additionally, analysis of the CGGA database indicated that high CKAP2 expression shortened the PFS of patients with HGG. The univariate and multivariate Cox regression analyses revealed that high CKAP2 expression may be an independent predictor for poor clinical outcomes in patients with HGG. Assessment of the association between CKAP2 expression and biological processes indicated that CKAP2 and its associated genes were enriched in the cell cycle, mitosis and cell proliferation in patients with glioma. The present study also demonstrated that CKAP2 may act as a biomarker in glioma progression and has the potential as a novel target for the treatment of HGG. However, further studies are required to identify the potential mechanisms by which CKAP2 regulates glioma cell biological functions.

The in vitro experiments further confirmed the key role of CKAP2 in the cell cycle and cell proliferation of glioma. Notably, the U-87MG and U-118MG cell lines have been reported to be contaminated, and are not the originally established cell lines $(32,33)$. However, U-87MG was confirmed to be derived from GBM and is still one of the most commonly used cell lines in glioma research (34-36). The American Type Culture Collection website declares that U-118MG and $\mathrm{U}-138 \mathrm{MG}$ are very similar cell lines, whereas they are not the same (https://www.atcc.org/Products/All/HTB-15. aspx\#characteristics). Furthermore, since both U-118MG and U-138MG glioma cell lines are derived from malignant gliomas, these misidentifications should not change the interpretation of the present results.

In conclusion, the present study revealed that CKAP2 was closely associated with tumor grade and could serve as an independent prognostic marker for OS and PFS in HGG. Furthermore, the results indicated that CKAP2 may act as an oncogene that promotes glioma tumor growth by activating the cell cycle, mitosis and cell proliferation pathways. However, the association between clinical features and CKAP2 expression was not fully elucidated. It is therefore necessary to further study the expression pattern and functional role of CKAP2 in the progression of glioma.

\section{Acknowledgements}

Not applicable.

\section{Funding}

The present study was funded by the National Nature Science Foundation of China (grant nos. 81502495 and 81702460).

\section{Availability of data and materials}

The datasets generated and/or analyzed during the current study are available in the CGGA repository (http://www.cgga. org.cn/index.php?m=Page\&a=index\&id=42).

\section{Authors' contributions}

TJ and HH designed the experiments. RH, GL and FZ analyzed the data and contributed analytical tools. $\mathrm{KW}, \mathrm{ZZ}$ and $\mathrm{YL}$ performed the experiments. $\mathrm{RH}$ and $\mathrm{KW}$ wrote the paper. All authors read and approved the final manuscript.

\section{Ethics approval and consent to participate}

The present study was approved by the Beijing Tiantan Hospital institutional review board (Beijing, China) and informed consent was obtained from all individual participants included in the study. 


\section{Patient consent for publication}

Consent was obtained from all individual participants included in the study.

\section{Competing interests}

The authors declare that they have no competing interests.

\section{References}

1. Jiang T, Mao Y, Ma W, Mao Q, You Y, Yang X, Jiang C, Kang C, Li X, Chen L, et al: CGCG clinical practice guidelines for the management of adult diffuse gliomas. Cancer Lett 375: 263-273, 2016.

2. Ricard D, Idbaih A, Ducray F, Lahutte M, Hoang-Xuan K and Delattre JY: Primary brain tumours in adults. Lancet 379: 1984-1996, 2012.

3. Louis DN, Perry A, Reifenberger G, von Deimling A, Figarella-Branger D, Cavenee WK, Ohgaki H, Wiestler OD, Kleihues P and Ellison DW: The 2016 World Health Organization classification of tumors of the central nervous system: A summary. Acta Neuropathol 131: 803-820, 2016.

4. Stupp R, Brada M, van den Bent MJ, Tonn JC and Pentheroudakis G; ESMO Guidelines Working Group: High-grade glioma: ESMO clinical practice guidelines for diagnosis, treatment and follow-up. Ann Oncol, 2014

5. Demuth T and Berens ME: Molecular mechanisms of glioma cell migration and invasion. J Neurooncol 70: 217-228, 2004.

6. Yan H, Parsons DW, Jin G, McLendon R, Rasheed BA, Yuan W, Kos I, Batinic-Haberle I, Jones S, Riggins GJ, et al: $I D H 1$ and IDH2 mutations in gliomas. N Eng J Med 360: 765-773, 2009.

7. Mellai M, Annovazzi L, Senetta R, Dell'Aglio C, Mazzucco M, Cassoni P and Schiffer D: Diagnostic revision of 206 adult gliomas (including 40 oligoastrocytomas) based on ATRX, IDH1/2 and 1p/19q status. J Neurooncol 131: 213-222, 2017.

8. Brandes AA, Franceschi E, Tosoni A, Benevento F, Scopece L Mazzocchi V, Bacci A, Agati R, Calbucci F and Ermani M Temozolomide concomitant and adjuvant to radiotherapy in elderly patients with glioblastoma: Correlation with MGMT promoter methylation status. Cancer 115: 3512-3518, 2009.

9. Wang J, Su HK, Zhao HF, Chen ZP and To SS: Progress in the application of molecular biomarkers in gliomas. Biochem Biophys Res Commun 465: 1-4, 2015.

10. Huse JT and Aldape KD: The evolving role of molecular markers in the diagnosis and management of diffuse glioma. Clin Cancer Res 20: 5601-5611, 2014.

11. Hong KU, Choi YB, Lee JH, Kim HJ, Kwon HR, Seong YS, Kim HT, Park J, Bae CD and Hong KM: Transient phosphorylation of tumor associated microtubule associated protein (TMAP)/cytoskeleton associated protein 2 (CKAP2) at Thr-596 during early phases of mitosis. Exp Mol Med 40: 377-386, 2008

12. Case CM, Sackett DL, Wangsa D, Karpova T, McNally JG Ried T and Camps J: CKAP2 ensures chromosomal stability by maintaining the integrity of microtubule nucleation sites. PLoS One 8: e64575, 2013.

13. Hong KU, Kim E, Bae CD and Park J: TMAP/CKAP2 is essential for proper chromosome segregation. Cell Cycle 8: 314-324, 2009.

14. Yoo BH, Kang DS, Park CH, Kang $\mathrm{K}$ and Bae CD: CKAP2 phosphorylation by $\mathrm{CDK} 1 /$ cyclinB1 is crucial for maintaining centrosome integrity. Exp Mol Med 49: e354, 2017.

15. Kim YW, Eom BW, Kook MC, Kim HS, Kim MK, Hwang HL, Chandra V, Poojan S, Song Y, Koh JS, et al: Clinical implications of proliferation activity in T1 or T2 male gastric cancer patients. Exp Mol Med 47: e193, 2015.

16. Gao Y, Liu X, Li T, Wei L, Yang A, Lu Y, Zhang J, Li L, Wang S and Yin F: Cross-validation of genes potentially associated with overall survival and drug resistance in ovarian cancer. Oncol Rep 37: 3084-3092, 2017.

17. Kim HS, Koh JS, Choi YB, Ro J, Kim HK, Kim MK, Nam BH, Kim KT, Chandra V, Seol HS, et al: Chromatin CKAP2, a new proliferation marker, as independent prognostic indicator in breast cancer. PLoS One 9: e98160, 2014.
18. Guo QS, Song Y,Hua KQ and Gao SJ: Involvement of FAK-ERK2 signaling pathway in CKAP2-induced proliferation and motility in cervical carcinoma cell lines. Sci Rep 7: 2117, 2017.

19. Viticchie G, Lena AM, Latina A, Formosa A, Gregersen LH, Lund AH,Bernardini S, Mauriello A, Miano R, Spagnoli LG, et al: MiR-203 controls proliferation, migration and invasive potential of prostate cancer cell lines. Cell Cycle 10: 1121-1131, 2011.

20. Yu G, Lee YC, Cheng CJ, Wu CF, Song JH, Gallick GE, Yu-Lee LY, Kuang J and Lin SH: RSK promotes prostate cancer progression in bone through ING3, CKAP2, and PTK6-mediated cell survival. Mol Cancer Res 13: 348-357, 2015.

21. Hayashi T, Ohtsuka M, Okamura D, Seki N, Kimura F, Shimizu H, Yoshidome H, Kato A, Yoshitomi H, Furukawa K, et al: Cytoskeleton-associated protein 2 is a potential predictive marker for risk of early and extensive recurrence of hepatocellular carcinoma after operative resection. Surgery 155: 114-123, 2014.

22. Yan W, Zhang W, You G, Zhang J, Han L, Bao Z, Wang Y, Liu Y, Jiang C, Kang C, et al: Molecular classification of gliomas based on whole genome gene expression: A systematic report of 225 samples from the Chinese Glioma Cooperative Group. Neuro Oncol 14: 1432-1440, 2012.

23. Bao ZS, Chen HM, Yang MY, Zhang CB, Yu K, Ye WL, Hu BQ, Yan W, Zhang W, Akers J, et al: RNA-seq of 272 gliomas revealed a novel, recurrent PTPRZ1-MET fusion transcript in secondary glioblastomas. Genome Res 24: 1765-1773, 2014.

24. Livak KJ and Schmittgen TD: Analysis of relative gene expression data using real-time quantitative PCR and the $2^{-\Delta \Delta C_{\mathrm{T}}}$ method. Methods 25: 402-408, 2001.

25. Hänzelmann S, Castelo R and Guinney J: GSVA: Gene set variation analysis for microarray and RNA-seq data. BMC Bioinformatics 14: 7, 2013.

26. Asbaghi Y, Thompson LL, Lichtensztejn $\mathrm{Z}$ and McManus KJ: KIF11 silencing and inhibition induces chromosome instability that may contribute to cancer. Genes Chromosomes Cancer 56: 668-680, 2017

27. Parrilla A, Cirillo L, Thomas Y, Gotta M, Pintard L and Santamaria A: Mitotic entry: The interplay between Cdk1, Plk1 and Bora. Cell Cycle 15: 3177-3182, 2016.

28. De Carli E, Wang X and Puget S: IDHI and IDH2 mutations in gliomas. N Eng J Med 360: 2248; author reply 2249, 2009.

29. DeAngelis LM: Brain tumors. N Eng J Med 344: 114-123, 2001.

30. Tsuchihara K, Lapin V, Bakal C, Okada H, Brown L, Hirota-Tsuchihara M, Zaugg K, Ho A, Itie-Youten A, Harris-Brandts M, et al: Ckap2 regulates aneuploidy, cell cycling, and cell death in a p53-dependent manner. Cancer Res 65: 6685-6691, 2005.

31. Kikuchi A, Fumoto K and Kimura H: The Dickkopf1cytoskeleton-associated protein 4 axis creates a novel signalling pathway and may represent a molecular target for cancer therapy. Br J Pharmacol 174: 4651-4665, 2017.

32. Capes-Davis A, Theodosopoulos G, Atkin I, Drexler HG, Kohara A, MacLeod RAF, Masters JR, Nakamura Y, Reid YA, Reddel RR and Freshney RI: Check your cultures! A list of cross-contaminated or misidentified cell lines. Int J Cancer 127: $1-8,2010$.

33. Allen M, Bjerke M, Edlund H, Nelander S and Westermark B: Origin of the U87MG glioma cell line: Good news and bad news. Sci Transl Med 8: 354re353, 2016.

34. Li H, Chen L, Li JJ, Zhou Q, Huang A, Liu WW, Wang K, Gao L, Qi S and Lu YT: $m i R-519 a$ enhances chemosensitivity and promotes autophagy in glioblastoma by targeting STAT3/Bcl2 signaling pathway. J Hematol Oncol 11: 70, 2018.

35. Jantas D, Grygier B, Golda S, Chwastek J, Zatorska J and Tertil M: An endogenous and ectopic expression of metabotropic glutamate receptor 8 (mGluR8) inhibits proliferation and increases chemosensitivity of human neuroblastoma and glioma cells. Cancer Lett 432: 1-16, 2018.

36. Guo B, Sheng Z, Hu D, Li A, Xu S, Manghnani PN, Liu C, Guo L, Zheng $\mathrm{H}$ and Liu B: Molecular engineering of conjugated polymers for biocompatible organic nanoparticles with highly efficient photoacoustic and photothermal performance in cancer theranostics. ACS Nano 11: 10124-10134, 2017.

This work is licensed under a Creative Commons Attribution-NonCommercial-NoDerivatives 4.0 International (CC BY-NC-ND 4.0) License. 